\title{
Classification of Intraoperative Complications: Reply
}

\author{
Salome Dell-Kuster ${ }^{1,2} \cdot$ Pierre-Alain Clavien $^{3} \cdot$ Heiner C. Bucher ${ }^{1} \cdot$ \\ Rachel Rosenthal ${ }^{4}$
}

Published online: 22 August 2015

(C) Société Internationale de Chirurgie 2015

We would like to thank Kaafarani and Velmahos [1] for their interest in our publication [2] and for pointing out to us their manuscript "Derivation and Validation of a Novel Severity Classification for Intraoperative Adverse Events" (iAE), published in June 2014 in the Journal of the American College of Surgeons [3] and further publications associated to the classification. We read with great interest about this newly derived classification system for surgical intraoperative adverse events.

Although the intention of the iAE classification system of intraoperative complications by Kaafarani and colleagues shares some common features with the CLASSIC system proposed by our group, the two classification systems differ in many ways. In CLASSIC, our interdisciplinary group of surgeons, anesthesiologists, epidemiologists, and statisticians aims at the development of a system reporting patient-related intraoperative complications including all events, regardless whether they are surgery- or anesthesia-related deviations from the ideal intraoperative course [2]. This approach is in accordance with the classification for postoperative complications by Clavien and Dindo, in which any deviation from the

Rachel Rosenthal

rachel.rosenthal@unibas.ch

1 Basel Institute for Clinical Epidemiology and Biostatistics, University Hospital Basel, Basel, Switzerland

2 Department of Anesthesiology, University Hospital Basel, Basel, Switzerland

3 Department of Surgery, University Hospital Zurich and University of Zurich, Zurich, Switzerland

4 Department of Surgery, University Hospital Basel, Basel, Switzerland normal postoperative course is captured $[4,5]$. We thus performed a Delphi process among 40 experts in various surgical subspecialties and methodological fields in 14 countries to develop this comprehensive classification system. The pilot validation based on a retrospective review of surgery, anesthesia, and pre- and postoperative records showed a high interrater agreement with an intraclass correlation coefficient of $83 \%$ (95\% confidence interval (CI) $73,90 \%$ ). A prospective multicenter international cohort study will look for the validation and further refinement of CLASSIC.

The proposed iAE classification system by Kaafarani et al., on the other hand, performed a Delphi process among seven general surgeons from a single center and focused only on intraoperative surgical adverse events [3]. As the authors state, this is important to enhance standardized reporting, allowing for comparison of the quality of intraoperative surgical care between different surgeons and hospitals. The validation relies on a retrospective analysis of the American College of Surgeons NSQIP database, administrative data, and operative reports relating to intra-abdominal and abdominal wall surgery, specifically on interventions coded with "Accidental Puncture or Laceration." We believe that the results of this validation are indeed encouraging and we are very interested to learn more about any further validation in a prospective and broader setting.

By focusing not only on surgical, but on any intraoperative complications, we assume that the CLASSIC system might perform better in predicting postoperative morbidity and mortality.

In conclusion, we welcome any system for structured and standardized reporting of complications and any collaboration. We believe that these two systems are distinct 
in their scope of adverse events to be captured and thereby may be important in different settings.

\section{Compliance with ethical standards}

Conflict of interest Rachel Rosenthal is an employee of F. Hoffmann-La Roche Ltd. since May 1, 2014. The CLASSIC study was conducted before she joined F. Hoffmann-La Roche Ltd. and has no connection to her employment by the company.

\section{References}

1. Kaafarani HMA, Velmahos GC (2015) Classification of intraoperative complications. World J Surg. doi:10.1007/s00268-015$3119-0$
2. Rosenthal R, Hoffmann H, Clavien PA et al (2015) Definition and classification of intraoperative complications (CLASSIC): Delphi study and pilot evaluation. World J Surg 39:1663-1671. doi:10. 1007/s00268-015-3003-y

3. Kaafarani HM, Mavros MN, Hwabejire J et al (2014) Derivation and validation of a novel severity classification for intraoperative adverse events. J Am Coll Surg 218:1120-1128

4. Clavien PA, Barkun J, de Oliveira ML et al (2009) The ClavienDindo classification of surgical complications: five-year experience. Ann Surg 250:187-196

5. Dindo D, Demartines N, Clavien PA (2004) Classification of surgical complications: a new proposal with evaluation in a cohort of 6336 patients and results of a survey. Ann Surg 240:205-213 